infliximab treatment. In reviewing the literature we find that anti TNF paradoxically brings about an immediate response in erythema nodosum patients, however provokes erythema nodosum and others skin manifestations in patients with either rheumatic pathology or inflammatory bowel disease. [1,2]

Bibliography

1. Clayton TH et al, Treatment of chronic erythema nodosum with infliximab. Clin Exp Dermatol. 2006 Nov; 31(6):823-4.

2. Ortego-Centeno $\mathrm{N}$ et al, Refractory chronic erythema nodosum successfully treated with adalimumab. J Eur Acad Dermatol Venereol. 2007 Mar; 21(3):408-10.

\section{Genetics and epigenetics of rheumatic diseases}

\section{A7.1 A GENETIC VARIANT IN THE REGION OF MMP-9 IS ASSOCIATED WITH SERUM LEVELS AND PROGRESSION OF JOINT DAMAGE IN RHEUMATOID ARTHRITIS}

doi:10.1136/annrheumdis-2013-203221.1

${ }^{1} \mathrm{DPC}$ de Rooy, 'A Zhernakova, ${ }^{2} \mathrm{R}$ Tsonaka, ${ }^{1} \mathrm{~A}$ Willemze, ${ }^{1} \mathrm{BAS}$ Kurreeman, ${ }^{1} \mathrm{REM}$ Toes, 'TWJ Huizinga, ${ }^{2} \mathrm{JJ}$ Houwing-Duistermaat, ${ }^{3} \mathrm{PK}$ Gregersen, ${ }^{1} \mathrm{AHM}$ van der Helm-van Mil. 'Department of Rheumatology, Leiden University Medical Center, Leiden, The Netherlands; 'Department of Medical Statistics, Leiden University Medical Center, Leiden, The Netherlands; ${ }^{3}$ Feinstein Institute for Medical Research and North ShoreLong Island Jewish Health System, Manhasset, New York, USA

Background and Objectives The severity of joint destruction is highly variable between Rheumatoid Arthritis (RA) patients. We aimed to identify new genetic risk factors by studying genetic susceptibility loci of several auto-immune diseases.

Patients and Methods In phase-1, 646 Dutch RA-patients with yearly X-rays of hands and feet over 7 years follow-up were genotyped for 148,880 SNPs by Immunochip which contains 186 loci previously associated with autoimmune diseases. Association of SNPs with MAF > 0.01 (130,841 SNPS) with joint destruction was analysed using a marginal regression model. Correction for multiple testing was done by Bonferroni correction for the number of uncorrelated SNPs (threshold $p<1.1 \times 10^{-6}$ ). In phase-2, 686 North American RA-patients with repeated hands X-rays over 15 years follow-up, for which Immunochip genotyping data were also available, were studied. SNPs that were significantly associated in phase- 1 were selected and evaluated. All X-rays were scored by Sharp van der Heijde score (ICC 0.91 and 0.98 for phase- 1 and 2 respectively). MMP-9 levels were measured in baseline serum by ELISA (Ebioscience) in 120 RA-patients that were selected on the Rs11908352-genotype.

Results In phase-1, 109 SNPs were significantly associated with joint destruction $\left(p<1.1 \times 10^{-6}\right)$. Of these, 76 variants were on the HLA region. The 33 non-HLA genetic variants, though several were in high LD, were studied in the North-American RA-patients. Here, after correction for the number of uncorrelated SNPs (threshold $p<0.0036$ ), two variants were associated with the severity of joint destruction: Rs451066 on chromosome 14 ( $p_{\text {uncorrected }}=0.002$, $\mathrm{MAF}=0.20)$ and Rs11908352 on chromosome 20 ( $p_{\text {uncorrected }}=0.002$, $\mathrm{MAF}=0.21$ ). The region of Rs451066 on chromosome 14 has previously been linked to type-1 diabetes susceptibility. Presence of a risk allele was associated with a $3.7 \%$ higher rate of joint destruction per year; this equaled 29\% over 7-years. Rs11908352 is located at the MMP-9 region on chromosome 20. Patients with a risk allele had a $2.7 \%$ higher radiological progression rate per year, which equaled $20 \%$ more joint destruction over a 7 -years period. Furthermore, the minor genotype was associated with significantly higher levels of MMP-9 compared to the common genotype ( $p=0.007)$.
Conclusions Two new risk loci for progressive joint destruction in RA were identified (Rs451066 and Rs1190852). The risk allele in Rs11908352 also associated with higher serum MMP-9 levels, indicating to a role for MMP-9 in progression of joint destruction in RA. Acknowledgements RACI study group, Dutch Arthritis Foundation, Netherlands organisation for scientific research.

\section{A7.2 ALLOGRAFT INFLAMMATORY FACTOR 1 (AIF1) POLYMORPHISMS IN FRENCH CAUCASIANS WITH RHEUMATOID ARTHRITIS}

doi:10.1136/annrheumdis-2013-203221.2

'Doua F Azzouz, 1,2Nathalie Balandraud, 'Sami B Kanaan, 'Isabelle Auger, 'Marielle Martin, 'Fanny Arnoux, ${ }^{1,2}$ Jean Roudier, 'Nathalie C Lambert. 'INSERM UMRS 1097, Marseille; ${ }^{2}$ Rheumatology Department, Ste Marguerite Hospital, AP-HM, Marseille

Background Allograft inflammatory factor 1 (AIF1) is a cytoplasmic inflammatory protein encoded within the HLA class III genomic region on chromosome 6 (6p21.3). Although several risk loci for Rheumatoid Arthritis (RA) have been identified by Genome Wide Association Studies (GWAS), none of them involved AIF1 polymorphisms. However, two studies on small cohorts have shown that AIF1 single nucleotide polymorphism (SNP) Rs2269475 (C/T), causing a non-synonymous change of amino acid, is associated with RA (Harney, SM et al, 2008; Pawlik A et al, 2008). Moreover, AIF1 overexpression in inflammatory synovial tissues and macrophages isolated from synovial fluids of patients with RA, confirms its potential role in RA.

Objective We propose to examine the association of the seven most described AIF1 SNPs in our French RA patients.

Methods We have tested 99 Anti-Citrullinated Protein Antibody (ACPA) positive Caucasian RA patients who fulfilled ACR/EULAR criteria and 104 healthy Caucasians. We designed AIF1 primers to specifically amplify the AIF1 gene region containing the 7 SNPs: Rs2844475, Rs4711274, Rs2736182, Rs2736181, Rs2259571, Rs2269475 and Rs13195276. PCR products were sequenced (Cogenics Beckman Coulter) and chromatogram results analysed for the 7 SNPs positions in patients and controls. Patients and controls were genotyped for HLA-DRB1.

Results Two SNPs out of the 7 were associated with RA: Rs4711274 (G/A) and Rs2269475 (C/T). Regarding Rs4711274, G/A and A/A genotypes were increased when compared with controls $(p=0.0005)$. The minor A allele was strongly associated with RA $(p=0.0005)$. Regarding Rs22699475, in linkage disequilibrium with the former, we found a similar pattern with increased $\mathrm{T} / \mathrm{T}$ and $\mathrm{C} / \mathrm{T}$ genotypes $(p=0.0009)$ and increased minor $T$ allele frequency $(p=0.0008)$ in patients with RA. Interestingly, patients carrying the minor associated AIF1 allele expressed HLA-DRB*04 more often than the patient's group carrying the $\mathrm{C} / \mathrm{C}$ or $\mathrm{G} / \mathrm{G}$ genotype $(63.8 \%$ versus $44.4 \%$ ), although the difference was marginal ( $p=0.06)$.

Conclusions In this study of French Caucasians with RA, we confirmed Rs2269475 association already described in British and Polish Caucasians. Additionally, we find an association with Rs4711274 in linkage disequilibrium with Rs2269475. Intriguingly, such associations have never been found in GWAS

\section{A7.3 ASSOCIATION OF CIRCULATING MIR-223 AND MIR-16 WITH DISEASE ACTIVITY IN PATIENTS WITH EARLY RHEUMATOID ARTHRITIS}

doi:10.1136/annrheumdis-2013-203221.3

'Mária Filková, 'Caroline Ospelt, 'Serena Vettori, 'Ladislav Šenolt, ${ }^{2}$ Heřman Mann, ${ }^{2} \mathrm{Jiřl}$ Vencovský, ${ }^{2}$ Karel Pavelka, 'Beat A Michel, ${ }^{1}$ Renate E Gay, ${ }^{1}$ Steffen Gay, ${ }^{1}$ Astrid Jüngel. ${ }^{1}$ Center of Experimental Rheumatology, University Hospital Zurich, Zurich, Switzerland; 2Institute of Rheumatology, Department of Clinical and Experimental Rheumatology of the $1^{\text {st }}$ Faculty of Medicine, Charles University in Prague, Prague, Czech Republic 